\title{
Outcomes following abdominal sacrocolpopexy for pelvic organ prolapse
}

\author{
Anushree Rawat*, Ruchika Garg, Poonam Yadav
}

Department of Obstetrics and Gynaecology, Sarojini Naidu Medical College, Agra, Uttar Pradesh, India

Received: 21 September 2020

Revised: 30 October 2020

Accepted: 02 November 2020

\section{*Correspondence:}

Dr. Anushree Rawat,

E-mail: anushree.jain10@gmail.com

Copyright: (c) the author(s), publisher and licensee Medip Academy. This is an open-access article distributed under the terms of the Creative Commons Attribution Non-Commercial License, which permits unrestricted non-commercial use, distribution, and reproduction in any medium, provided the original work is properly cited.

\section{ABSTRACT}

Background: Hysterectomy is one of the most performed surgical procedures during lifetime. Almost $10 \%$ of women who have had a hysterectomy because of prolapse symptoms. Sacrocolpopexy (sacral colpopexy) is a surgical technique to repair pelvic organ prolapses. Specifically, it is intended to address apical or vaginal vault prolapse in women with lower perioperative morbidity, shorter hospital stay and allows a long-term anatomical restoration. It provides excellent apical support with lower rate of recurrence. Aim of present study was to assess the efficiency of conventional method of sacrocolpopexy and to assess the follow up complaints of patients.

Methods: This study is conducted in maternal and child health wing of Sarojini Naidu medical college from June 2019 till December 2019. Sacrospinous fixation was performed with conventional needle holder as compared to Miya hook. Data were collected from participants using a structured questionnaire. Follow up was conducted at 6 weeks and 12 weeks.

Results: In this study there were $43.3 \%$ pre menopausal patients and $53.6 \%$ menopausal patients. Maximum patients were multiparous i.e. para $2(36.6 \%)$. Maximum patient belongs to POP Q stage 3. After 6 weeks of surgery maximum patients had pain in abdomen i.e. $3.33 \%$, dyspareunia $8.33 \%$ and $100 \%$ success rate of sacropexy.

Conclusions: Sacrocolpopexy is a cost effective and safe procedure with high anatomical cure and patient satisfaction rate and low intra-operative and. postoperative complications as well as recurrence rates.

Keywords: Vault prolapse, Pelvic organ prolapse, Sacrocolpopexy, Sacrospinous fixation/suspension, Conventional needle holder

\section{INTRODUCTION}

Hysterectomy is one of the most performed surgical procedures during lifetime. Almost $10 \%$ of women, who have had a hysterectomy because of prolapse symptoms, will visit a gynaecologist for a surgical correction of a vaginal vault prolapse thereafter. Surgical treatment should be offered to women with symptomatic PHVP after appropriate counselling as per RCOG guideline in 2015. A cochrane review comparing abdominal sacrocolpopexy to vaginal sacrospinous fixation considered the open abdominal procedure as the treatment of first choice for prolapse of the vaginal vault, although operation time and hospital stay is longer. ${ }^{1}$ The type of operation performed should be tailored to the individual patient's circumstances.

Sacrocolpopexy (sacral colpopexy) is a surgical technique to repair pelvic organ prolapses. Specifically, it is intended to address apical or vaginal vault prolapse in women. The salient component of reconstruction is suspension of the apical portion of vagina (or the vaginal cuff in patients after hysterectomy) in a manner that recreates the natural anatomic support that the uterosacral and cardinal ligaments provide, usually by tacking it to the sacral promontory. Sacrospinous fixation (SSF) at the 
time of vaginal hysterectomy should be considered when the vault descends to the introitus during closure. ${ }^{2}$

Abdominal sacro colpopexy (ASC) is associated with significantly lower rates of recurrent vault prolapse, dyspareunia and postoperative stress urinary incontinence (SUI) when compared with sacrospinous fixation (SSF). However, this is not reflected in significantly lower reoperation rates or higher patient satisfaction. ${ }^{2} \mathrm{SSF}$ is associated with earlier recovery compared with ASC.

SSF may not be appropriate in women with short vaginal length and should be carefully considered in women with pre-existing dyspareunia. $^{2}$

\section{Objectives}

The objectives of current study were to assess the efficiency of conventional method of sacrocolpopexy and to assess the follow up complaints of patients.

\section{METHODS}

Presented study is a retrospective study of post operative patients was conducted in maternal and child health wing of Sarojini Naidu medical college from June 2019 till December 2019. Total 60 patients participated in the study and data was collected from participants using a structured questionnaire. Follow up was conducted at 6 weeks and 12 weeks.

The protocol and the effect of surgery were investigated by reviewing and analyzing the clinical data of 60 recurrent patients (grade and above), who had received the pelvic floor repair surgery from June 2019 to December 2019.

The extent of uterine or vaginal prolapse was assessed by gynaecological examination and POP quantification system (POP-Q) for prolapse assessment.

Follow up questionnaire about quality of life (pelvic floor distress inventory-short form 20 (PFDI-20) and pelvic floor impact questionnaire short form (PFIQ-7) were used to evaluate objective and subjective efficacy, respectively. $^{3}$

\section{Inclusion criteria}

Inclusion criteria for current study were; symptomatic uterine or vaginal vault prolapse patients with POP-Q stage 2 and above, symptoms include a sensation of pressure on the vagina and perineum, seeing and feeling of a bulge/protusion in the distal vagina, chronic pelvic pain, dyspareunia and other sexually related problems or associated lower urinary tract symptoms including urgency, frequency, urinary retention and incontinence.

\section{Exclusion criteria}

Inclusion criteria for current study were; patients having a contraindication for surgery, pelvic inflammatory disease, patients who had undergone pelvic radiotherapy, patients with compromised immune status interfering with recovery and patients who were lost after surgery for follow-up.

The procedure was performed after informed consent from the patient and is performed under anaesthesia using conventional 16 inch needle holder and prolene 1.0 suture for sacrospinous fixation as compared to Miya hook used for sacropexy.

\section{Statistical analysis}

The SPSS version 22.0 software program was used for statistical analysis.

\section{RESULTS}

Suggestive menstrual status of the patients who participated in the study is depicted in (Table 1). There were $43.3 \%$ pre menopausal patients and $56.6 \%$ menopausal patients.

Table 1: Case distribution according to menstrual status.

\begin{tabular}{|ll|}
\hline Menstrual status & N $(\%)$ \\
\hline Pre menopausal & $26(43.3)$ \\
\hline Post menopausal & $34(56.6)$ \\
\hline
\end{tabular}

The parity of the patients involved in the study is described in (Table 2). Maximum patients were multiparous i.e. para $2(36.6 \%)$, followed by para 3 $(25 \%)$ and para $1(16.6 \%)$. Only 5 patients were nulliparous.

Table 2: Case distribution according to parity.

\begin{tabular}{|ll|}
\hline Parity & $\mathbf{N}(\%)$ \\
\hline Nulliparous & $5(8.3)$ \\
\hline $\mathbf{1}$ & $10(16.6)$ \\
\hline $\mathbf{2}$ & $22(36.6)$ \\
\hline $\mathbf{3}$ & $15(25)$ \\
\hline$>\mathbf{3}$ & $8(13.3)$ \\
\hline
\end{tabular}

Various complaints of patients who underwent surgery is described in (Figure 1). Maximum patients presented with the complaint of pelvic organ prolapse i.e. 28 in number followed by 22 patients with stress incontinence and 10 with urinary retention. 8 patients had bowel complaints like constipation and painful defecation. 17 patients had decreased sensation and loss of vaginal tone whereas 20 patients had heaviness feeling in lower abdomen. 
Patients who were selected for surgery after POP quatification is described in (Figure 2). Maximum patient belong to stage 3 i.e. 40 followed by 10 patients of stage 4 and 10 of stage 2 . No patient of stage 1 was selected for the surgery.

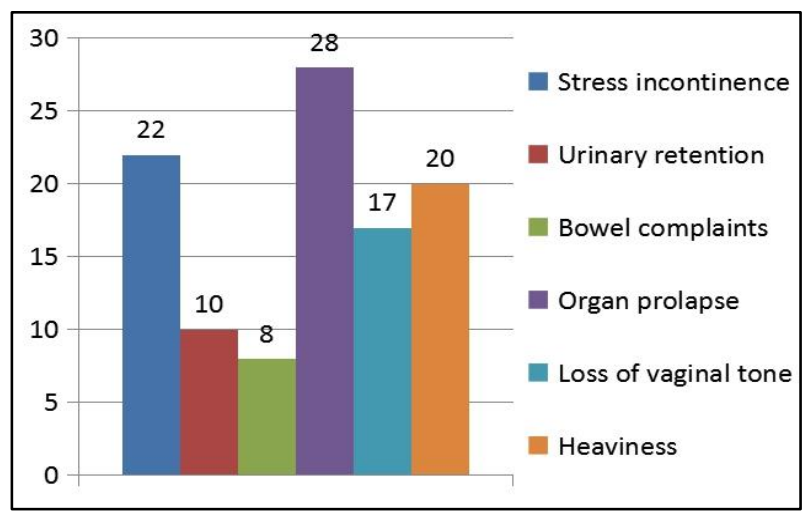

Figure 1: Case distribution according to patients complaints before surgery.

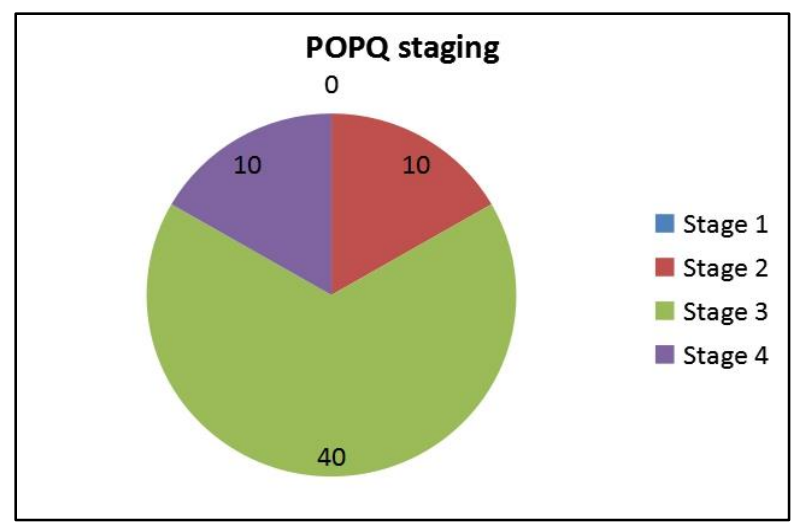

\section{Figure 2: Suggestive of patients selected for surgery} according to POPQ staging.

Follow up complaints observed in patients post 6 weeks and 12 weeks of surgery is described in (Table 3). As observed after 6 weeks maximum patients had pain in abdomen i.e. $3.33 \%$ cases followed by complaint of dyspareunia $(8.33 \%)$, pain during defecation $(6.66 \%)$, urinary complaints like retention in $1.66 \%$ and incontinence in $3.33 \%$ cases. Relapse of pelvic organ prolpse was seen in $3.33 \%$ of the patients.

Table 3: Results of follow up visits at 6 weeks and 12 weeks post-surgery.

\begin{tabular}{|l|l|l|}
\hline Post surgery complaints & 6 weeks & 12 weeks \\
\hline Pain in abdomen & $2(3.33 \%)$ & $1(1.6 \%)$ \\
\hline Bleeding per vaginum & $5(8.33 \%)$ & $2(3.33 \%)$ \\
\hline Urinary incontinence & $2(3.33 \%)$ & $2(3.33 \%)$ \\
\hline Urinary retention & $1(1.66 \%)$ & 0 \\
\hline Constipation & $2(3.33 \%)$ & $1(1.66 \%)$ \\
\hline Painful Defecation & $4(6.66 \%)$ & $1(1.66 \%)$ \\
\hline Dyspareunia & $5(8.33 \%)$ & $2(3.33 \%)$ \\
\hline Pelvic organ prolapse & $2(3.33 \%)$ & $1(1.66 \%)$ \\
\hline
\end{tabular}

At 12 weeks the major complaint was pain in abdomen in $1.66 \%$ cases followed by urinary incontinence and bleeding per vaginum in $3.33 \%$ and relapse of pelvic organ prolapse in $1.66 \%$ cases. Other contributing complaints were dysparenunia and constipation.

\section{DISCUSSION}

Abdominal sacrocolpopexy (ASC) is considered as excellent procedure in the surgical management of apical Prolapse. ${ }^{5}$ ASC has been superior to other techniques in terms of restroration of normal vaginal axis and maintenance of vaginal capacity. ${ }^{4-6}$ Sacrocolpopexy is a reliable procedure that effectively and consistently resolves vaginal vault prolapse.

In this study there were $43.3 \%$ pre menopausal patients and $53.6 \%$ menopausal patients. Maximum patients were multiparous i.e. para $2(36.6 \%)$ followed by para 3 $(25 \%)$, para $1(16.6 \%)$. Only 5 patients were nulliparous.

Patients who were selected for surgery after POP quatification. Maximum patient belong to stage 3 i.e. 40 followed by 10 patients of stage 4 and 10 of stage 2 . No patient of stage 1 was selected for the surgery.

Patients presented with the complaint of pelvic organ prolapse i.e. 28 in number followed by 22 patients with stress incontinence and 10 with urinary retention. 8 patients had bowel complaints like constipation and painful defecation. 17 patients had decreased sensation and loss of vaginal tone whereas 20 patients had heaviness feeling in lower abdomen.

The follow up complaints observed in patients post 6 weeks and 12 weeks of surgery. As observed after 6 weeks maximum patients had pain in abdomen i.e. $3.33 \%$ cases followed by complaint of dyspareunia (8.33\%), pain during defecation $(6.66 \%)$, urinary complaints like retention in $1.66 \%$ and incontinence in $3.33 \%$ cases. Relapse of pelvic organ prolpse was seen in $3.33 \%$ of the patients.

At 12 weeks the major complaint was pain in abdomen in $1.66 \%$ cases followed by urinary incontinence and bleeding per vaginum in $3.33 \%$ and relapse of pelvic organ prolapse in $1.66 \%$ cases. Other contributing complaints were dysparenunia and constipation.

Authors found $100 \%$ success rate of sacropexy, similar results were obtained by Rani et al, Nygaard et al, in the review of 2178 patients reported a success rate of 78$100 \%$. They reported $4.9 \%$ rate of stress urinary incontinence. $^{4}$

Weidner et al reported 2 cases of sacral osteomyelitis and hemorrhage from presacral veins had been reported in 1$2.6 \%$ patients. Higgs et al found $90 \%$ success rate with $3 \%$ recurrence rate on long term follow-up, $12 \%$ patient reported reduced vaginal capacity with dyspareunia and 
subject satisfaction rate was $78 \%$. In this study no major intraoperative and postoperative complications were encountered. $^{7-9}$

Sacrocolpopexy is a cost effective and safe procedure with high anatomical cure and patient satisfaction rate and low intra-operative and postoperative complications as well as recurrence rates.

\section{Limitations}

The study included limited ability to assess the improvement in PFDI-20 and PFIQ-7 scores between the pre- and postoperative status. A randomized controlled trial might add more information regarding the current results. However in order to reduce the interobsevation bias all the cases were evaluated and operated by the same team of surgeons and the follow up was also carried out by the same team.

\section{CONCLUSION}

Abdominal sacrocolpopexy is associated with a lower rate of recurrent vault prolapse and dyspareunia than the vaginal sacrospinous colpopexy. These benefits must be balanced against a longer operating time, longer time to return to activities of daily living and increased cost of the abdominal approach. The extent of recurrence, the recurrent site and complications must be carefully considered and evaluated for re-treatments of recurrence after pelvic floor repair surgery, and then an appropriately individualized re-treatment protocol could be designed for each of the patients. Sacrocolpopexy is a reliable procedure that effectively and consistently resolves vaginal vault prolapse and uterine prolapse with lower perioperative morbidity, shorter hospital stay and allows a long-term anatomical restoration. It provides excellent apical support with lower rate of recurrence.

Funding: No funding sources Conflict of interest: None declared

Ethical approval: The study was approved by the Institutional Ethics Committee

\section{REFERENCES}

1. Coolen AWM, van IJsselmuiden MN, van Oudheusden AMJ, Veen J, van Eijndhoven HWF, Mol BWJ, et al. Laparoscopic sacrocolpopexy versus vaginal sacrospinous fixation for vaginal vault prolapse, a randomized controlled trial: SALTO-2 trial, study protocol. Bio Med Cent Wom Heal. 2017; 17(1):52.

2. Post hsterectomy vaginal vault prolapsed. Available at: https://www.rcog.org.uk/en/guidelines-researchservices/guidelines/gtg46/. Accessed on 20 August 2020.

3. Barber MD, Walters MD, Bump RC. Short forms of two condition-specific quality-of-life questionnaires for women with pelvic floor disorders. Am J Obstet Gynecol. 2005; 193:103-8.

4. Fan SX, Wang FM, Lin LS, Song YF. Re-treatments of recurrence after pelvic floor repair surgery. Zhonghua Fu Chan Ke Za Zhi. 2017;52(6):374-8.

5. Nygaard I, McCreery R, Brubaker L. For the pelvic Floor disorders network. Abdominal Sacrocolpopexy: a comprehensive Review. Obstet Gynecol. 2004;104:805-23.

6. Lane FE. Repair of posthysterectomy vaginal-vault prolapse. Obstet Gynecol. 1962;20:72-7.

7. Grunberger W, Grunberger V, Wierrani F. Pelvic promontory fixation of the vaginal vault in sixty-two patients with prolapse after hysterectomy. J Am Coll Surg. 1994;178:69-72.

8. Rani S, Pandher DK, Huria A, Mehra R. Clinical outcome of abdominal sacrocolpopexy. J Midlife Health. 2015;6(4):169-72.

9. Higgs PL, Chua HL, Smith ARB. Long term review of laparoscopic sacrocolpopexy. Bri J Obstet Gynaecol. 2005;112:1134-8.

Cite this article as: Rawat A, Garg R, Yadav P. Outcomes following abdominal sacrocolpopexy for pelvic organ prolapse. Int J Reprod Contracept Obstet Gynecol 2020;9:4970-3. 